\title{
RESEÑA \\ XI Congreso Internacional de la AMIT "30 Años de Investigación Turística en México"
}

\author{
Irma Magaña Carrillo \\ Universidad de Colima \\ Maribel Osorio García \\ Universidad Autónoma del \\ Estado de México
}

E I congreso anual de la Academia Mexicana de Investigación Turística (AMIT) es un evento esperado con interés no solo por los miembros de esta asociación, sino también por estudiantes de licenciatura y posgrado de las diferentes carreras de turismo y áreas afines del país. EI XI Congreso Internacional de la Aмıт: “30 Años de Investigación Turística en México” se llevó a cabo del 27 al 29 de septiembre de 2017, el cual se realizó conjuntamente con la Universidad de Guadalajara, en el Centro Universitario de Ciencias Económico Administrativas (CUCEA).

La celebración del trigésimo aniversario del estudio científico del turismo en México se desarrolló con la participación de especialistas de diversas generaciones, propiciando el diálogo e intercambio de ideas, conocimientos y avances de investigaciones entre los académicos con el tema central de los adelantos del turismo en materia científica.

Este congreso dio muestra de una efervescencia de inquietudes para formar grupos, redes y nuevas oportunidades de colaboración con miras al futuro. Se constituye así en un espacio de encuentro donde entablar un diálogo entre actores de diferentes disciplinas y perspectivas de análisis, con objeto de reflexionar sobre los cambios, las condiciones y oportunidades para hacer investigación, y los espacios

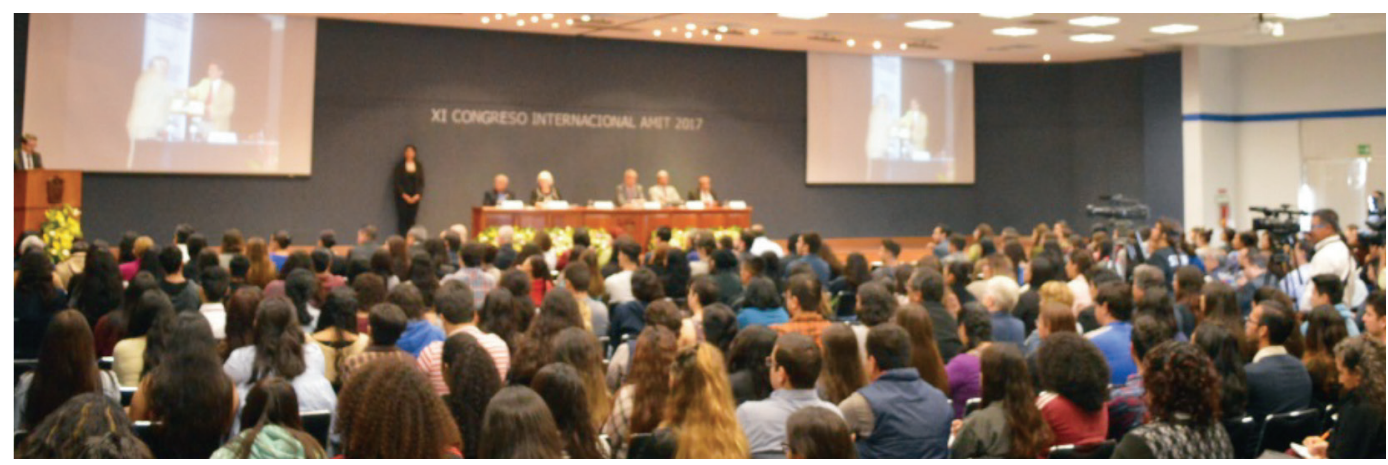

CÓMO CITAR: Magaña, I. y Osorio-García, M. (2018). Reseña del XI Congreso Internacional de la AMIT «30 Años de Investigación Turística en México». Dimensiones Turísticas, 2(2), 137-143. https://doi.org/10.47557/NSSM7220 

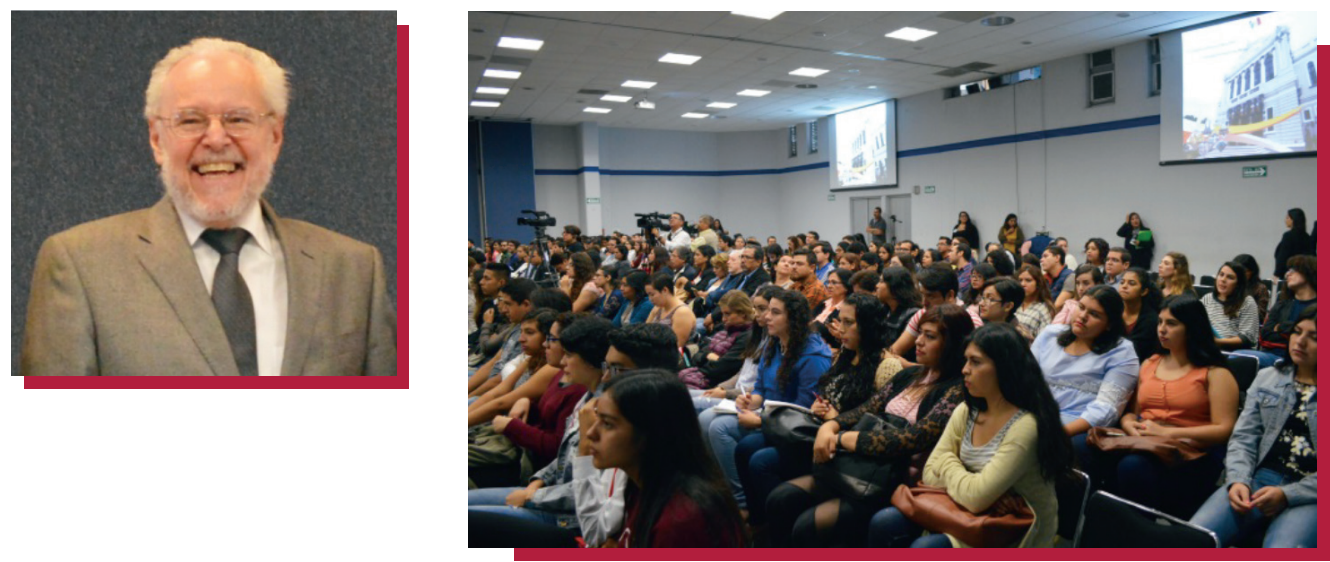

para establecer relaciones de colaboración entre los diversos participantes que son parte de la formación, diseño, ejecución, financiamiento, publicación y reconocimiento de los resultados de investigación en turismo.

El evento dio inicio con el curso pre-Congreso “El estudio científico de la sociedad”, impartido por el doctor Fernando Miguel Leal Carretero, destacado investigador de la Universidad de Guadalajara. Posteriormente, el 27 de septiembre se realizó la inauguración oficial del Congreso, con la participación de autoridades universitarias, gubernamentales y la presencia de la doctora Nora L. Bringas Rábago, actual presidenta de la AMIT. Durante la ceremonia se destacó la importancia y los beneficios que el turismo ha traído para el desarrollo del país, tanto en el ámbito económico como en el cultural, principalmente.

La inauguración fue enmarcada con dos interesantes eventos magistrales. Se abrió con una conferencia titulada “Las ciencias sociales ¿disciplinas o áreas de estudio?”, a cargo del doctor Leal Carretero, quien hizo una reflexión-análisis para clarificar la naturaleza del turismo como objeto de estudio y su riqueza multidisciplinaria.

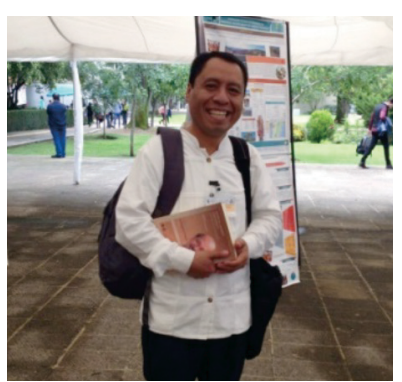

Después se desarrolló el panel "Investigación de la gastronomía: miradas multidisciplinarias", coordinado por la maestra Angélica Guerra Ávalos, y contó con la participación de Ricardo J. Ávila Palafox (Centro Universitario de Ciencias Sociales y Humanidades-Universidad de Guadalajara), Claudia Rocío Magaña González (Centro Universitario del Sur-Universidad de Guadalajara), Lourdes Flores López (Centro de Investigación y Asistencia en Tecnología y Diseño del Estado de Jalisco-Consejo Nacional de Ciencia y Tecnología), Nora L. Bringas Rábago (El Colegio de la Frontera Norte) y Marco Antonio Covarrubias Castro (Universidad del Claustro de Sor Juana). Sin duda, un panel interesante en el que cada uno de los participantes mostró su nivel de conocimiento en el área y coincidieron en que la gastronomía es un activo inseparable, figurando en muchas ocasiones como el principal motivo para regresar a un lugar.

El mismo día, en la explanada del auditorio central del CUCEA se expusieron los carteles participantes en el Congreso, que fueron alrededor de 20 sobre diversas temáticas. 


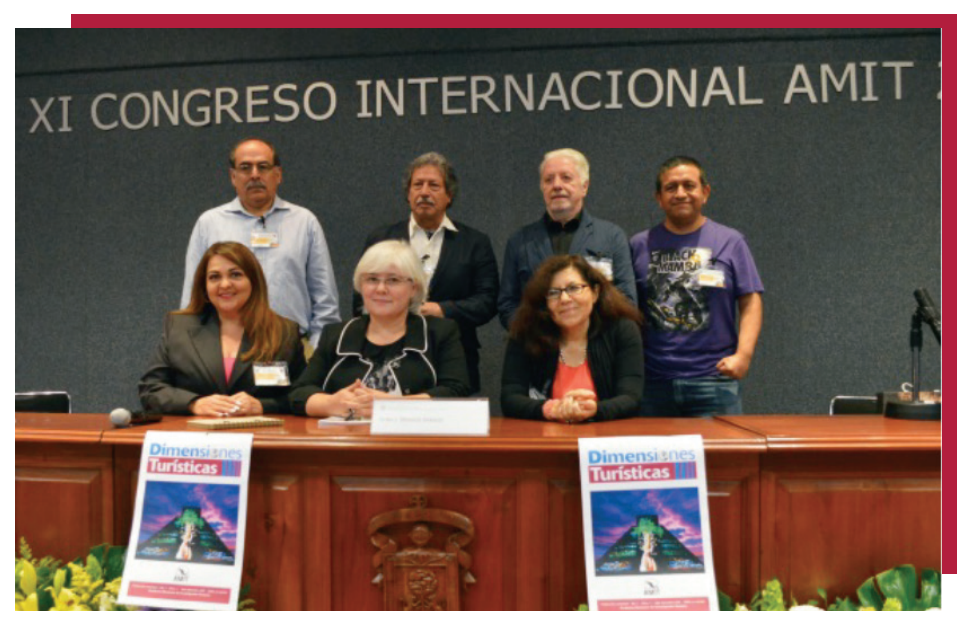

Uno de los momentos más emotivos y relevantes durante el Congreso Amit 2017 lo constituyó la presentación del primer número de la revista Dimensiones Turísticas, uno de los proyectos estratégicos que el actual Consejo de la AMIT se propuso concretar al inicio de su gestión. Esta revista tiene la intención de convertirse en un producto académico sobresaliente y viene a llenar un espacio importante de difusión entre quienes hacen investigación y generan nuevo conocimiento en el campo del turismo. Se reconoció que el camino no es fácil para llegar a ser una revista de calidad y posicionarse como una de las mejores en su género, pero se cuenta con el esfuerzo conjunto de los miembros de la academia para alcanzar el estatus que se exige en estos casos y poder ingresar en los más prestigiados índices de impacto nacionales e internacionales.

Por la tarde del miércoles 28 y el jueves 29 de septiembre se inició la presentación de 139 ponencias en 24 mesas de trabajo que versaron sobre epistemología; capacitación y competitividad; educación y tecnologías de la información; gestión de conocimiento turístico; empleo turístico; planificación de destinos; evaluación de impactos en destinos turísticos; producción del espacio turístico; percepciones sociales; gestión territorial; turismo, sustentabilidad y cambio climático; cultura e identidad; y patrimonio turístico, entre otros. Adicionalmente hubo tres mesas temáticas: La formación de investigadores en los posgrados de turismo en México; Turismo y centros históricos: ¿oportunidades o amenazas?; y Reflexión desde la atractividad territorial de Jalisco como destino turístico, de inversiones y capital humano. En total participaron 170 expositores de 35 universidades nacionales de diferentes puntos cardinales del país y de cuatro universidades extranjeras (Argentina, Brasil, Colombia y Ecuador). Los resultados, propuestas y experiencias generados durante el encuentro reflejan el avance de la investigación turística en México, pero también en Latinoamérica.

Asimismo, durante el segundo día del Congreso se realizó un panel con el tema: "30 años de investigación turística en México”, en el cual fungió como moderador el doctor Basilio Verduzco Chávez (CUCEA-Universidad de Guadalajara) y participaron los doctores Daniel Hiernaux-Nicolas (Universidad Autónoma de Querétaro), Alfonso Jiménez Martínez 
(Universidad Autónoma de Guadalajara), Felipe Cuamea Velázquez (Universidad Autónoma de Baja California), Salvador Gómez Nieves (CuCEA-Universidad de Guadalajara) y Carlos Rogelio Virgen Aguilar (Centro Universitario de la Costa-Universidad de Guadalajara). Los panelistas vertieron sus experiencias y dificultades en el camino hacia un trabajo investigativo para estudiar el turismo mexicano que a la fecha ha dado frutos, enfatizando la necesidad de seguir en esa vía para asegurar el desarrollo del conocimiento del turismo.

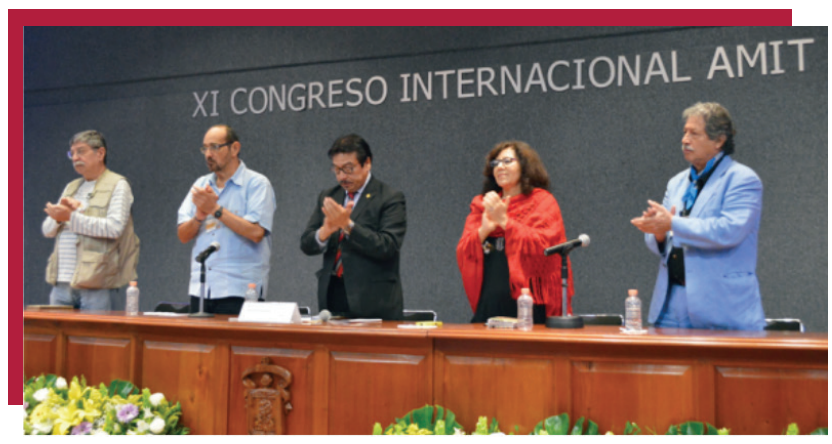

Dentro de las actividades que se llevaron a cabo, hubo dos homenajes post mórtem. El primero para el doctor Sergio Molina Espinoza, cuyo moderador fue el doctor Tomás de Jesús Cuevas Contreras, de la Universidad Autónoma de Ciudad Juárez. Los participantes en este foro fueron la doctora Noemí Wallingre (Universidad Nacional de Quilmes, Argentina), el maestro Jorge Trujillo Rincón (Universidad Autónoma de Chiapas), el doctor Marcelino Castillo Néchar (Universidad Autónoma del Estado de México), el maestro Fernando Mandri Bellot (Integra Tourism for Good), el doctor Roger Bergeret Muñoz (Universidad Autónoma de Guerrero), el doctor Carlos Virgen Aguilar (Centro Universitario de la Costa-Universidad de Guadalajara) y la doctora Maribel Osorio García (Universidad Autónoma del Estado de México). Todos ellos expresaron su relación personal con el maestro Sergio Molina, destacando su calidad humana y resaltando la trascendencia de sus investigaciones y sus valiosos aportes a la investigación turística en México.

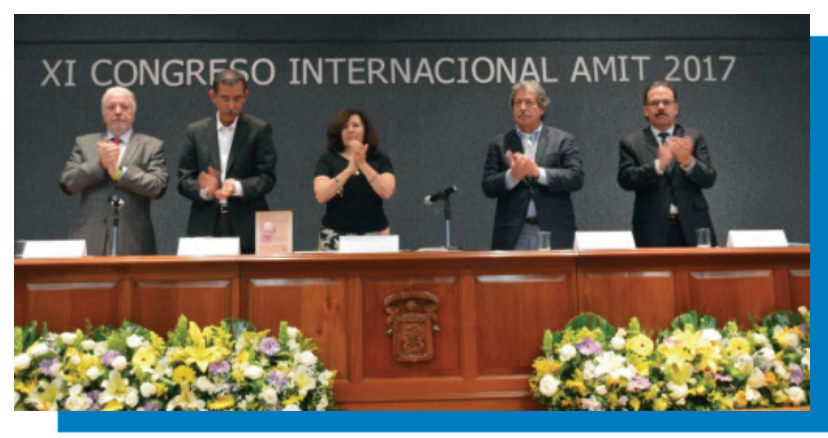

El segundo homenaje fue para el licenciado Manuel Rodríguez Woog, el cual moderó la doctora Maribel Osorio García (Universidad Autónoma del Estado de México) y en el que participaron los doctores Felipe Cuamea Velázquez (Universidad Autónoma de Baja California), Daniel Hiernaux-Nicolas (Universidad Autónoma de Querétaro), Alfonso de Jesús Jiménez (Universidad Autónoma de Guadalajara) y el maestro José de Jesús Quiñónez Ramírez (Instituto de Competitividad Turística-Secretaría de Turismo). Los participantes también compartieron con el público asistente la calidad humana del homenajeado y su importante trayectoria y aportaciones a la investigación del turismo. Vale la pena destacar que estos dos personajes fueron pioneros en el estudio del turismo en México. 
En el marco del Congreso también se presentaron los siguientes libros: Desarrollo de destinos turísticos consolidados: una visión desde la sustentabilidad y la resiliencia (2017), de A. Montaño Armendáriz, A. Ivanova Boncheva y R. Serrano Castro (coords.); Guía introductoria a la dimensión temática del sistema turístico, Acapulco (2017), de A. J. Jiménez Martínez, D. D. Ortiz Guzmán, M. Velarde Valdez y R. J. Bergeret Muñoz; Experiencias de turismo rural en Quintana Roo (2017), de J. F. Domínguez Estrada; Herramientas conceptuales y metodológicas para la gestión turística (2016), de J. G. Ruiz Andrade; e Internacionalización de los servicios de salud. Turismo médico en México y Jalisco (2016), de J. Guzmán Flores.

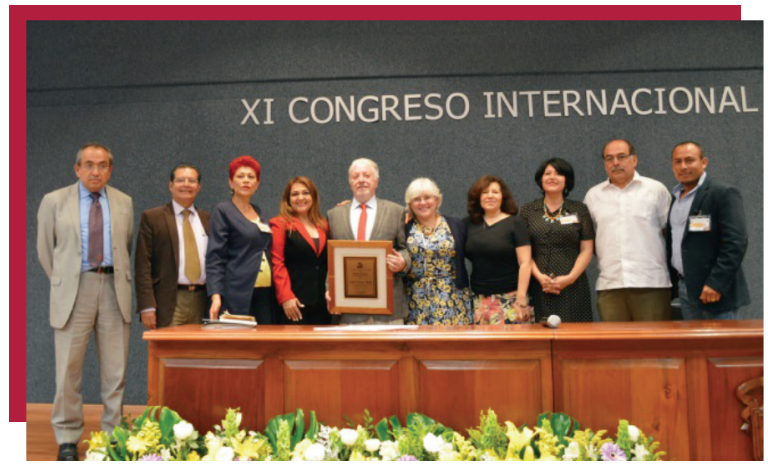

Finalmente, con la intención de reconocer los esfuerzos realizados por estudiantes y miembros de la comunidad científica del turismo, la AmIT otorgó el Premio Nacional a la Trayectoria de Investigación Turística 2017, el cual se entrega anualmente a un investigador, considerando su trayectoria y contribución al conocimiento científico del turismo; y el Premio Nacional a la Mejor Tesis de Posgrado 2016 en dos categorías: Maestría y Doctorado.

El Premio Nacional a la Trayectoria de Investigación Turística 2017 fue otorgado al doctor Daniel Hiernaux-Nicolas (Universidad Autónoma de Querétaro), tras la deliberación realizada por el Comité Evaluador, conformado por los doctores José Fernando Vera Rebollo y Raquel Huete Nieves (Univer-

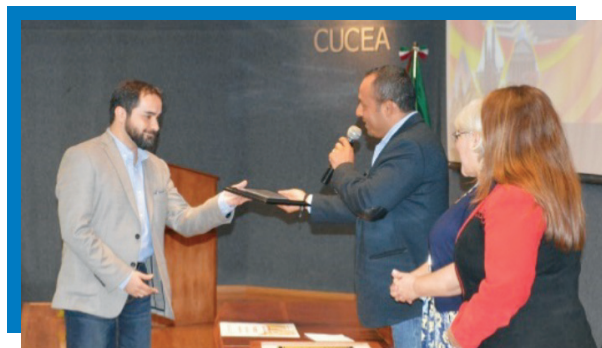

Dr. Federico Gerardo Zúñiga Bravo, Premio Nacional a la Mejor Tesis de Posgrado 2016, categoría Doctorado.

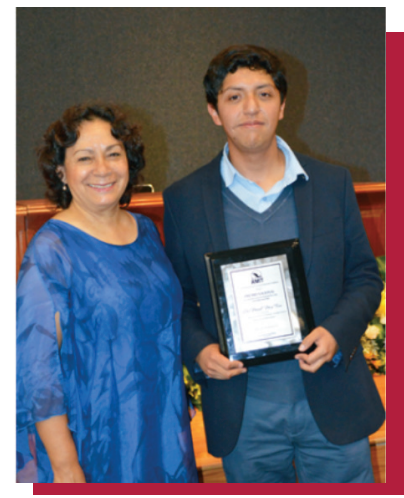

Mtro. Víctor Manuel Mora Torres, Premio Nacional a la Mejor Tesis de Posgrado 2016, categoría Maestría, y su directora, Dra. Rocio Serrano Barquin. paña),Agutín Santana Talavera (Universidad de La Laguna, España), Basilio Chávez Verduzco (CUCEA-Universidad de Guadalajara) y Maribel Osorio García (Universidad Autónoma del Estado de México). Asimismo, el Premio Nacional a la Mejor Tesis de Posgrado 2016, categoría Maestría, fue entregado a Víctor Manuel Mora Torres por su trabajo intitulado Turismo religioso en el Cerrito 


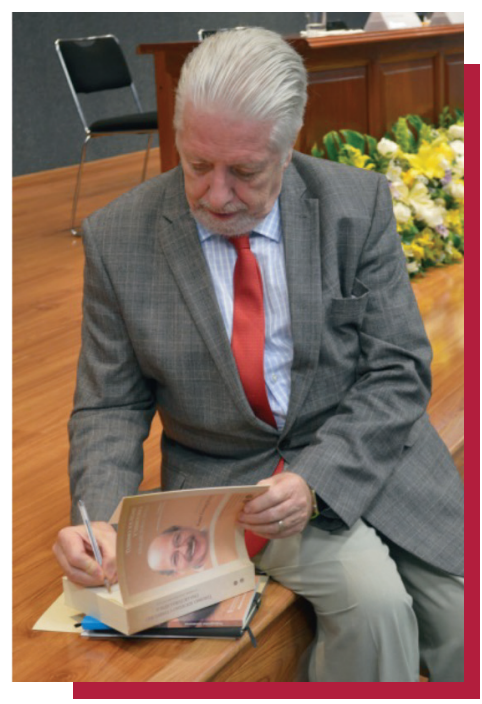

Dr. Daniel Hiernaux-Nicolas, Premio Nacional a la Trayectoria de Investigación Turística 2017.

de Tepexpan: un análisis desde el enfoque de la desdiferenciación, mientras que el de categoría Doctorado fue para Federico Gerardo Zúñiga Bravo, de la Universidad Nacional Autónoma de México, por su investigación Patrimonio cultural, etnicidad y turismo. Procesos de patrimonialización, turistificación y mercantilización en el Totonacapan veracruzano.

La última conferencia magistral, "El turismo del siglo xIX: cerrar/abrir puertas para su estudio" estuvo a cargo del recién galardonado, doctor Daniel Hiernaux-Nicolas, en la cual trasladó a los asistentes a los orígenes de la investigación turística y planteó sus vicisitudes, retos, logros y fracasos, develando un camino largo y sinuoso del quehacer investigativo en materia de turismo. El conferencista destacó la relevancia de investigaciones que marcaron interesantes aportes al campo del conocimiento del turismo y principalmente a la actividad turística.

El 29 de septiembre este magno evento llegó a su fin, su realización fue una buena oportunidad para ampliar los nexos entre la investigación turística y otras miradas científicas sobre el turismo y sus manifestaciones. Un aspecto que estuvo presente a lo largo del Congreso fue la necesidad de mantener vigente la relación entre la docencia, la investigación y la publicación de resultados. Como producto de este puente se destacó la trascendencia de la aparición de la revista Dimensiones Turísticas, la presentación de libros, el reconocimiento a la trayectoria en investigación turística y a las tesis de posgrado, y el intercambio de resultados de investigación.

Otro aspecto que prevaleció durante todo el Congreso fue la necesidad de fortalecer un modelo de colaboración intergeneracional para mantener viva la memoria de quienes han contribuido con su conocimiento al desarrollo de la investigación turística de México, de ahí la relevancia de reconocer sus aportaciones. Esto resulta significativo porque recupera la experiencia y muestra una visión del futuro que se quiere construir para la investigación turística. Igualmente se resaltó la importancia de reconocer el papel de los jóvenes como creadores del conocimiento en un contexto mediado por lo tecnológico, en lo social, ambiental, cultural y político, situación que estuvo fuera del alcance de quienes hacían investigación hace 30 años y que seguramente en el futuro cercano potenciará más el análisis del turismo como objeto de estudio.

Todo este trabajo académico estuvo aderezado por actividades socioculturales organizadas por los anfitriones. El primer día se brindó un coctel de bienvenida en el Museo de Artes de Zapopan y un video mapping en Plaza de las Américas, ambos por parte de la Dirección de Turismo de Zapopan, Jalisco. El segundo día se ofreció un recorrido nocturno en el Tapatío Tour por Guadalajara y Zapopan, bajo el auspicio de la Secretaría de Turismo del 


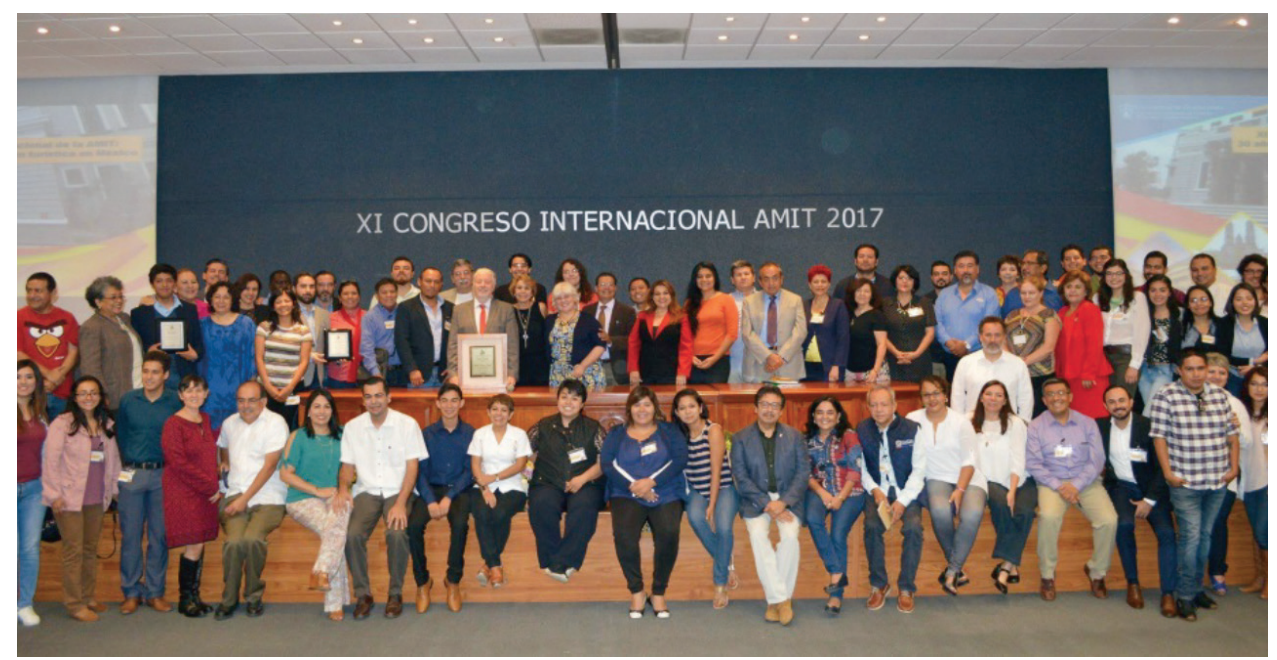

Estado de Jalisco. Para la clausura se realizó una cena de gala mexicana en el restaurante El Patio, tradicional sitio en Tlaquepaque, Jalisco, ofrecida por la Oficina de Visitantes y Convenciones de Guadalajara.

Por otra parte, se informó que el Congreso Amı 2018 se llevará a cabo en Cancún, Quintana Roo, con el auspicio de la Universidad del Caribe, gracias a la disposición de su rectora, la doctora Ana Pricila Sosa Ferreira. Por último, se efectuó la clausura de este notable encuentro, donde estuvieron presentes miembros del Consejo de la AMIT y autoridades de la Universidad de Guadalajara.

Cabe mencionar que el XII Congreso de la AMIT se celebrará de manera conjunta con el VII Congreso Latinoamericano de Investigación Turística (CLAIT) y con el XVI Seminario de Turismo y Sustentabilidad de la Universidad del Caribe, por lo que la AMIT, desde el cierre del Congreso 2017, ya se encuentra trabajando arduamente para la versión 2018, en espera de reunir especialistas de todo el continente para analizar el turismo desde diferentes perspectivas, con miras a generar una discusión creativa que permita avanzar en el entendimiento y comprensión del turismo como objeto de estudio. 